\title{
Simplified calculations of band-gap renormalization in quantum-wells
}

\author{
K. GüVEn, B. TANATAR \\ Department of Physics, Bilkent University, Bilkent, 06533 Ankara, Turkey
}

(Received 3 January 1996)

\begin{abstract}
Non-linear optical properties of photoexcited semiconductor quantum-wells are of interest because of their opto-electronic device application possibilities. Many-body interactions of the optically created electrons and holes lead to the band-gap renormalization which in turn determines the absorption spectra of such systems. We employ a simplified approach to calculate the band-gap renormalization in quantum-well systems by considering the interaction of a single electron-hole pair with the collective excitations (plasmons). This method neglects the exchange-correlation effects but fully accounts for the Coulomb-hole term in the single-particle self-energy. We demonstrate that the density, temperature, and well-width dependence of the band-gap renormalization for GaAs quantum-wells within our model is in good agreement with the experimental results.
\end{abstract}

(C) 1996 Academic Press Limited

Non-linear optical properties of photoexcited semiconductor quantum-wells are of interest because of their opto-electronic device application possibilities. Band-gap renormalization arising from the many-body interactions of optically created electrons and holes is an important ingredient to understand the absorption spectra of such systems. Screening in the electron-hole system leads to a renormalization of the single-particle energies. In particular, the Coulomb interaction between the carriers results in a decrease in the average charge density felt by individual particles. These manybody interactions along with the Pauli exclusion principle reduce the energy of charge carriers in valence and conduction bands. The narrowing of the band-gap affects the luminescence properties with interesting consequences for the semiconductor lasers [1].

The full many-body calculations of the band-gap renormalization make use of the perturbation theory to calculate the electron and hole self-energies at the conduction and valence band edges. The contribution to the self-energy may be split into a screened-exchange and a Coulomb-hole term. The former is calculated using the screened Coulomb potential in which various models and approximations for the dielectric function is employed. The Coulomb-hole term, on the other hand, describes the charge-density fluctuations around individual carriers. The general conclusions drawn from numerous studies [2-4] are such that for bulk materials the band-gap renormalization exhibits a universal density dependence [5], whereas the quantum-well systems show marked dependence on the well-width.

The main purpose of this communication is to extend the recent calculations of Ninno et al. [6] to quantum-well systems, and explore the well-width and temperature dependence of the band-gap renormalization to make more realistic contact with experiments. We demonstrate that the simple approach of calculating the band-gap renormalization, which neglects the exchange-correlation effects but fully accounts for the Coulomb-hole contribution, yields reasonable agreement with experi- 
mental results, provided well-width and temperature dependences are included. This is chiefly due to the fact that the Coulomb-hole part of the self-energy becomes dominant for not too high densities, as also noted by Haug and Schmitt-Rink [2]. Similar model calculations [7] based on the Coulombhole contribution have proven quite useful in bulk systems. Microscopic calculations [8-10] taking the finite well-width and temperature dependence for quasi-two-dimensional (Q2D) systems are generally in good agreement with the experimental results [11-13]. Various simplified approaches [14-16] for $3 \mathrm{D}$ and $2 \mathrm{D}$ systems provide understanding for the basic mechanisms of band-gap renormalization.

In the following, we calculate the energy of a free electron-hole pair interacting only with plasmons for a Q2D system. We include the well-width and finite temperature dependences. The resulting band-gap renormalization shows satisfactory agreement with the experiments.

We express the Hamiltonian of an electron-hole pair interacting with the collective modes (plasmons) as [6,17].

$$
H=\sum_{i=\mathrm{e}, \mathrm{h}} \frac{P_{i}^{2}}{2 m_{i}}+\sum_{q} \omega_{q}\left(a_{q}^{\dagger} a_{q}+\frac{1}{2}\right)+\sum_{i=\mathrm{e}, \mathrm{h}} \sum_{q} M_{i}^{D}(q)\left(a_{q} e^{i \mathbf{q} \cdot \mathbf{r}}+a_{q}^{\dagger} e^{-i \mathbf{q} \cdot \mathbf{r}}\right),
$$

in which we have neglected the direct electron-hole Coulomb interaction (we take $\hbar=1$ ). In the above expression, $M_{i}^{D}(q)$ is the matrix element describing the interaction of $i$ th carrier (electron or hole) with plasmons for a $D$-dimensional system, and $\omega_{q}$ is the $q$-dependent plasma dispersion. Since this Hamiltonian is formally identical to that of the polaron problem, straightforward application of the Lee-Low-Pines variational scheme [18] gives

$$
E_{g}=-\sum_{i=\mathrm{e}, \mathrm{h}} \int \frac{d^{D} q}{(2 \pi)^{D}}\left|M_{i}^{D}(q)\right|^{2}\left(\omega_{q}+q^{2} /\left(2 m_{i}\right)\right)^{-1},
$$

for the energy of the electron-hole pair interacting with plasmons. The carrier-plasmon interaction matrix element for a $D$-dimensional system is found to be $\left|M_{i}^{D}(q)\right|^{2}=\left[V_{q}^{D}\right]^{2} N q^{2} /\left(2 m_{i} \omega_{q}\right)$, where $V_{q}^{D}$ is the Coulomb interaction. For a 3D system $V_{q}=4 \pi e^{2} /\left(\epsilon_{0} q^{2}\right)$, and for a $2 \mathrm{D}$ system $V_{q}=2 \pi e^{2} f(q) /\left(\epsilon_{0} q\right)$, where $\epsilon_{0}$ is the static dielectric constant of the semiconducting material. In the 2D Coulomb interaction, we have allowed for a form factor $f(q)$ arising from the subband quantization. An infinite square-well model with width $a$ yields

$$
f(q)=\frac{8}{(q a)^{2}+4 \pi^{2}}\left[\frac{3}{8} q a+\frac{\pi^{2}}{q a}-\frac{4 \pi^{4}}{(q a)^{2}} \frac{1-e^{-q a}}{(q a)^{2}+4 \pi^{2}}\right] .
$$

The plasma dispersion $\omega_{q}$ is evaluated within the static plasmon-pole approximation [17]

$$
\omega_{q}^{2}=\omega_{\mathrm{pl}}^{2}(q) \frac{\varepsilon(q)}{\varepsilon(q)-1}
$$

where $\varepsilon(q)$ is the static dielectric function (i.e. $\varepsilon(q)=\varepsilon(q, \omega=0)$ ). We use the random-phase approximation (RPA) at zero and finite temperature for $\varepsilon(q)$. (The explicit forms we use may be found in the references cited.) $\omega_{\mathrm{pl}}(q)$ is the long-wavelength limit of the plasma frequency which reads $\omega_{\mathrm{pl}}^{2}=4 \pi e^{2} N /\left(\epsilon_{0} \mu\right)$ and $\omega_{\mathrm{pl}}^{2}(q)=2 \pi e^{2} N q /\left(\epsilon_{0} \mu\right)$ for $3 \mathrm{D}$ and $2 \mathrm{D}$ systems, respectively. Here $\mu$ is the reduced mass of the electron-hole pair.

We now discuss our results for the case of GaAs, for which the material parameters are: $m_{e}=0.067 \mathrm{~m}, m_{\mathrm{h}}=0.62 \mathrm{~m}$, where $m$ is the bare electron mass, and $\epsilon_{0}=13.18$ (we use the same material parameters as Ref. [6]). The band-gap renormalization within the present approach in bulk GaAs at zero temperature was calculated by Ninno et al. [6]. It was found that the density dependence of $E_{g}$ was reasonably accounted for. We first explore the temperature dependence of $E_{g}$ in our 


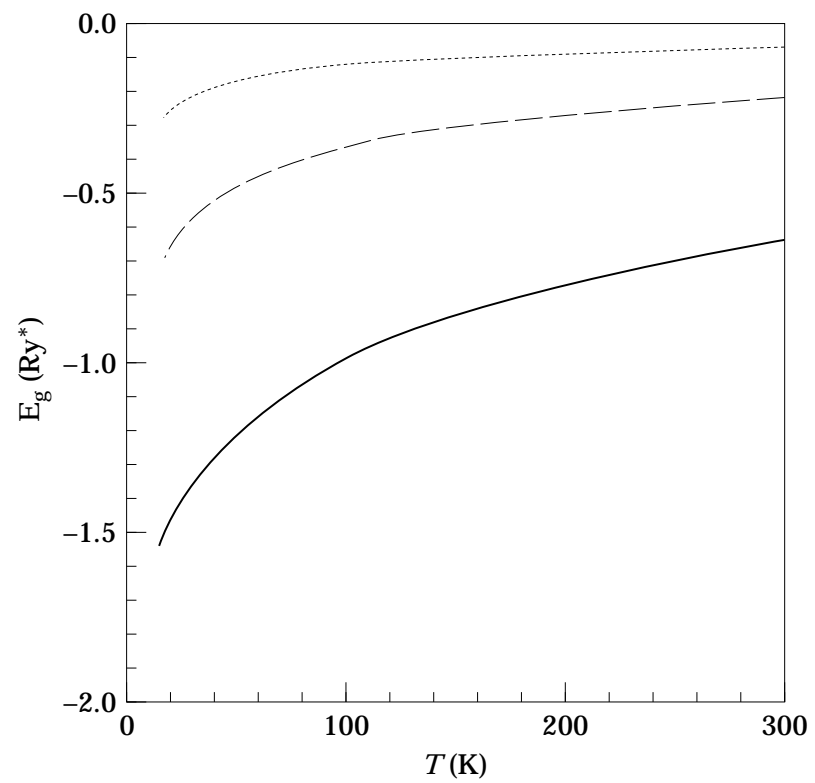

Fig. 1. Band-gap renormalization in bulk GaAs as a function of carrier temperature, for plasma densities $N=10^{14} \mathrm{~cm}^{-3}$ (dotted), $N=10^{15} \mathrm{~cm}^{-3}$ (dashed), and $N=10^{16} \mathrm{~cm}^{-3}$ (solid). We use the effective Rydberg for the energy scale, i.e. $\mathrm{Ry}^{*}=e^{2} \mu /\left(2 \epsilon_{0}^{2}\right)$.

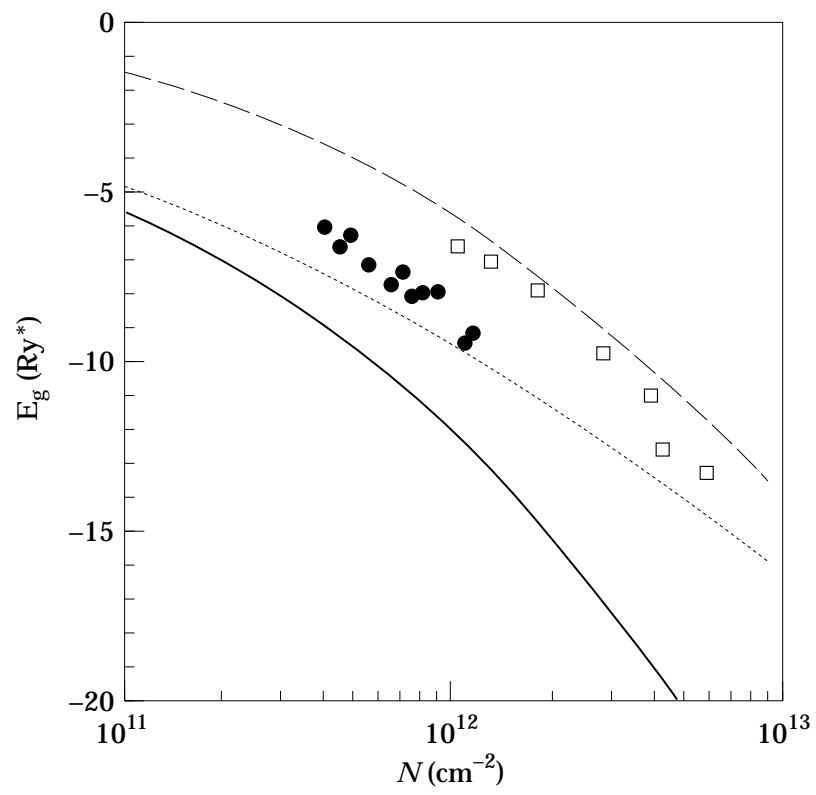

Fig. 2. Band-gap renormalization in $\mathrm{Q} 2 \mathrm{D} \mathrm{GaAs}$ as a function of plasma density. Solid line is for a strictly $2 \mathrm{D}$ system at $T=0$. Dotted and dashed lines indicate $E_{g}$ for a quantum-well of width $a=100 \AA$, at $T=0$ and $T=300 \mathrm{~K}$, respectively. The solid circles and open squares are the experimental data of Tränkle et al. [11] and Lach et al. [12], respectively. 


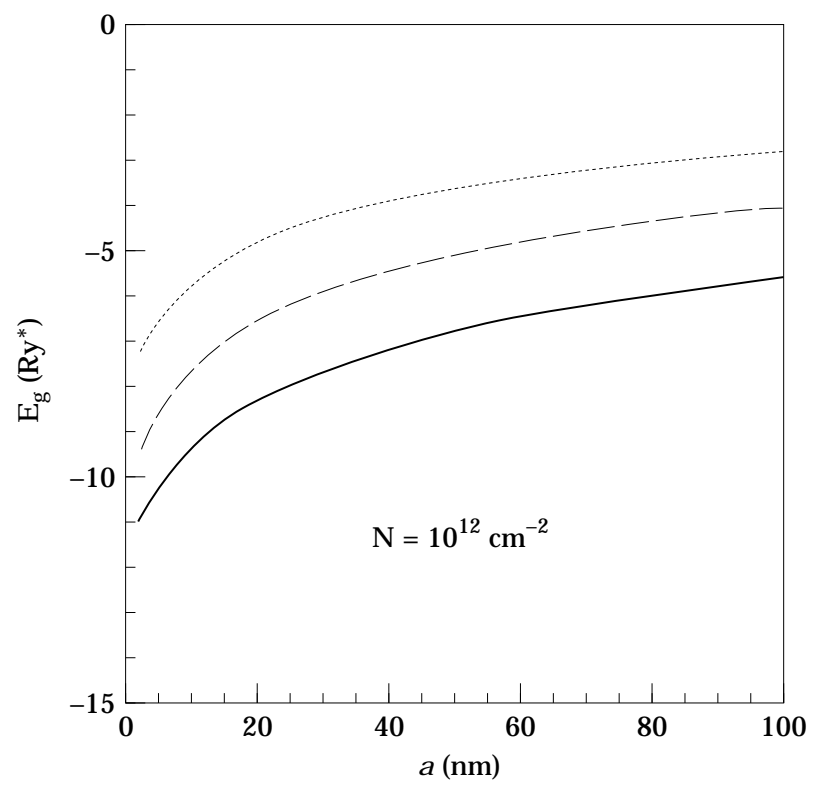

Fig. 3. Band-gap renormalization in $\mathrm{Q} 2 \mathrm{D} \mathrm{GaAs}$ as a function of the quantum-well width. Solid, dashed, and dotted lines indicate $T=0,100$, and $300 \mathrm{~K}$, respectively.

simplified scheme, for a 3D system. Figure 1 shows the band-gap renormalization in bulk GaAs as a function of carrier temperature for fixed plasma densities $N=10^{16} \mathrm{~cm}^{-3}$ (solid), $N=10^{15} \mathrm{~cm}^{-3}$ (dashed), and $N=10^{14} \mathrm{~cm}^{-3}$ (dotted). We observe that the temperature dependence of $E_{g}$ is stronger for lower plasma densities. In so far as the population of higher subbands is neglected, such behavior is also obtained in more detailed calculations [8].

The renormalized band-gap energy in a Q2D GaAs system as a function of the plasma density $N$ is depicted in Fig. 2. The solid line is for a strictly 2D electron-hole system at zero temperature. For comparison we also show the experimental data by Tränkle et al. [11] (solid circles) and Lach et al. [12] (open squares). The former of these measurements were taken on GaAs/GaAlAs samples of quantum-well-widths $21-83 \AA$, at $T=2 \mathrm{~K}$. The dotted line shows $E_{g}$ for a $100 \AA$ wide quantum-well at $T=0$, and the agreement with the data improves considerably. Lach et al. [12] data on the other hand, is for a $103 \AA \mathrm{GaAs} / \mathrm{GaAlAs}$ quantum-well at $300 \mathrm{~K}$, and cover a higher range of plasma densities. The dashed line shows $E_{g}$ calculated for a $100 \AA$ quantum-well at $300 \mathrm{~K}$ is also in reasonable agreement with the experimental data [12]. Our simplified model demonstrates the importance of including the finite well-width and temperature dependences in the band-gap renormalization. Calculations of Ryan and Reinecke [10] have already shown the importance of Coulomb-hole contribution over the screened-exchange in quantum wells for carrier densities of $N<3 \times 10^{12} \mathrm{~cm}^{-2}$.

Having identified the importance of well-width and temperature dependence of the band-gap renormalization, we show in Fig. 3, $E_{g}$ as a function of quantum-well width at a fixed 2D plasma density $N=10^{12} \mathrm{~cm}^{-2}$. The solid, dashed, and dotted lines are for $T=0,100$, and $300 \mathrm{~K}$, respectively.

In the above simplified approach of band-gap renormalization, we have considered the interaction of a single electron-hole pair with plasma excitations. It is well-known that a two-component system (i.e. electron-hole liquid) also supports an acoustic-plasmon mode [19] other than ordinary plasmons. We have attempted to include the contribution of acoustic modes to $E_{g}$ within the present approach, and found their effect to be rather small. This is due to their relatively weak interaction 
strengths, and limited region of existence in the phase-space [19]. The effect of carrier-phonon interactions on the band-gap renormalization was investigated by Das Sarma et al. [9] in a many-body formalism. In our case, the phonon contribution to screening is treated in the so-called $\epsilon_{0}$-approximation. We have also neglected the intersubband contribution to the self-energy within the present approach. Extension of the present approach to take for instance the intervalence band transitions into account should be straightforward once the dielectric function is suitably modified [10]. We have attempted to apply similar ideas for a quantum-well wire. In this case, we found that the Coulombhole term does not represent the experimental results well, suggesting the importance of screenedexchange term in the self-energy. Another possible source of discrepancy is our use of the bulk effective masses for the conduction and valence bands. In a confined system such as a quantum-well or a quantum-wire more accurate band masses should be used.

In summary, we have studied the band-gap renormalization in GaAs quantum-well systems within a simple model of electron-hole pair interacting with collective plasma excitations. We have found that experimentally observed band-gap energies may be accounted for qualitatively if the quantum-well width and temperature dependences are considered. Our calculations indicate that Coulomb-hole term is more important than the screened exchange term, in the self-energy calculations, and it represents the experimental data reasonably well.

Acknowledgements-This work is partially supported by the Scientific and Technical Research Council of Turkey (TUBITAK) under Grant No. TBAG-AY/77.

\section{References}

[1] H. Haug and S. W. Koch, Phys. Rev. A 39, 1887 (1989).

[2] H. Haug and S. Schmitt-Rink, Prog. Quantum Electron. 9, 3 (1984).

[3] H. Kalt and M. Rinker, Phys. Rev. B 45, 1139 (1992).

[4] G. Tränkle, Phys. Rev. B 36, 6712 (1987); Phys. Rev. Lett. 58, 419 (1987).

[5] P. Vashista and R. K. Kalia, Phys. Rev. B 25, 6492 (1982).

[6] D. Ninno, F. Liguori, V. Cataudella and G. Iadonisi, J. Phys. Condens. Matter 6, 9335 (1994).

[7] R. Zimmermann, M. Roesler and V. M. Asnin, Phys. Stat. Sol. (b) 90, 175 (1981); J. P. Loewenau, S. Schmitt-Rink and H. Haug, Phys. Rev. Lett. 49, 1511 (1982).

[8] R. Zimmermann, E. H. Bötcher, N. Kirstaedler and D. Bimberg, Superlatt. Microstruct. 7, 433 (1990).

[9] S. Das Sarma, R. Jalabert and S.-R. Eric-Yang, Phys. Rev. B 41, 8288 (1990).

[10] J. C. Ryan and T. L. Reinecke, Phys. Rev. B 47, 9615 (1993); Superlatt. Microstruc. 13, 177 (1993).

[11] G. Tränkle, E. Lach, A. Forchel, F. Scholz, C. Ell, H. Haug, G. Weimann, G. Griffiths, H. Kroemer and Subbanna, Phys. Rev. B 36, 6712 (1987).

[12] E. Lach, A. Forchel, D. A. Broido, T. L. Reinecke, G. Weimann and W. Schlapp, Phys. Rev. B 42, 5395 (1990).

[13] G. Bongiovanni and J. L. Staehli, Phys. Rev. B 39, 8359 (1989); C. Weber, C. Klingshirn, D. S. Chemla, D. A. B. Miller, J. E. Cunningham and C. Ell, ibid. 38, 12748 (1988); V. D. Kulakovskii, E. Lach, A. Forchel and D. Grützmacher, ibid. 40, 8087 (1989).

[14] F. Thuselt, Phys. Lett. A 94, 93 (1983).

[15] C. Ell, R. Blank, S. Benner and H. Haug, J. Opt. Soc. Am. B 6, 2006 (1989).

[16] S. Schmitt-Rink and C. Ell, J. Lumin. 30, 585 (1985).

[17] A. W. Overhauser, Phys. Rev. B 3, 1888 (1971).

[18] T. D. Lee, F. Low and D. Pines, Phys. Rev. 90, 297 (1953).

[19] G. Vignale and K. S. Singwi, Solid State Commun. 44, 259 (1982); G. Vignale, Phys. Rev. B 38, 811 (1988). 